\title{
Common ash (Fraxinus excelsior L.) in Ukrainian forests and its successful natural regeneration
}

\author{
Viktor Tkach ${ }^{1}$, Maksym Rumiantsev ${ }^{1}$, Volodymyr Luk'yanets ${ }^{1}$, \\ Oleksii Kobets ${ }^{1,}{ }^{*}$, Svitlana Pozniakova ${ }^{2}$, Iryna Obolonyk ${ }^{1}$ and \\ Serhii Sydorenko ${ }^{1}$
}

Tkach, V., Rumiantsev, M., Luk'yanets, V., Kobets, O., Pozniakova, S., Obolonyk, I., Sydorenko, S. 2020. Common ash (Fraxinus excelsior L.) in Ukrainian forests and its successful natural regeneration. - Forestry Studies | Metsanduslikud Uurimused 73, 26-42, ISSN 14069954. Journal homepage: http:/ / mi.emu.ee/forestry.studies

\begin{abstract}
The natural distribution area of common ash (Fraxinus excelsior L.) in the forests of Europe and Ukraine has been determined through the analysis of scientific works of Ukrainian and foreign researchers. According to the reference materials on the current state of Ukrainian forests ( 25 administrative regions), it is established that ash forests cover an area of about 150,000 ha or $2.4 \%$ of the total area of the country's forests. Among them, common ash stands account for almost $87 \%$ or 130,000 ha; the rest are stands of introduced ash species, namely F. pennsylvanica Marsh., F. americana L., and F. lanceolata Borkh. The average age of ash stands is 59 years. Only $6.4 \%(8,300 \mathrm{ha})$ of the total area of ash forests is covered by pure ash stands, and the remaining area $(93.6 \%$ or $121,700 \mathrm{ha})$ are mixed ones. It is revealed that the optimal natural and climatic conditions for the emergence, preservation and successful growth of natural regeneration of ash trees are fresh and moist fertile sites (rich in minerals) with well-drained soils, the light of at least $5-6 \%$ of that on an open space, and without abundant live ground cover. The highest number of seed regeneration of ash, about 7,000-8,000 stems ha- 1 on average, was found under the canopy of mixed (ash share in the composition was 10 to $20 \%$ by stock) old (over 100 years old) stands with a relative density of stocking of $0.7-0.8$. Promoting the natural seed regeneration of ash trees can be an effective way of increasing the sustainability of such stands and will ensure their preservation in the forests of Ukraine and in deciduous forests of Europe as a whole.
\end{abstract}

Key words: stand composition, stand age, occurrence frequency.

Author's addresses: ${ }^{1}$ Ukrainian Research Institute of Forestry and Forest Melioration named after G. M. Vysotsky, 86, Pushkinska Street, 61024, Kharkiv, Ukraine; ${ }^{2}$ Kharkiv National Agrarian University named after V. V. Dokuchajev, „Dokuchaevske-2”, 62483, Kharkiv district, Kharkiv region, Ukraine; "e-mail: alexei_kobec@ukr.net

\section{Introduction}

Common ash (Fraxinus excelsior L.) is one of the 30 main forest-forming species in the forests of Ukraine (State, 2016). In the country, ash stands grow on an area of about 150,000 hectares, as of 2012, or cover $2.4 \%$ of the total forest area (Gulchak, 2012). Among them, nearly 130,000 hectares $(87 \%)$ are dominated by common ash; the remaining area is covered by stands with introduced ash species (F. pennsylvan-

DOI: $10.2478 /$ fsmu-2020-0012

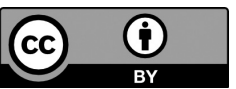

(C) 2020 by the authors. Licensee Estonian University of Life Sciences, Tartu, Estonia. This article is an open access article distributed under the terms and conditions of the Creative Commons Attribution (CC BY) license (http:// creativecommons.org/licenses/by/4.0/). 
ica Marsh., F. americana L., and F. lanceolata Borkh. (Matsiakh \& Kramarets, 2014). This area is significant because, for example, in Greece, the ash area is only 200 hectares or $1.5 \%$ of the country's forest area (Boiko et al., 2012); it is 14,000 hectares or almost $1 \%$ of the total forest area in Latvia (Liepins et al., 2016), 15,400 hectares or $0.4 \%$ in Belarus (Domnenkov et al., 2014), 19,200 hectares or $10 \%$ in Ireland (Wellock et al., 2014), about 40,000 hectares or $1.4 \%$ in the Czech Republic (Giagli et al., 2018), about 90,000 hectares or $1.0 \%$ in Poland (Boiko, 2007), 250,000 hectares or $2.4 \%$ of the total forest area in Germany (Enderle et al., 2017).

Ash stands, occupying a unique ecological niche, are valuable not only as a source of high-quality wood and by-products, but also as a powerful regulator of biosphere processes, an accumulator of huge carbon deposits, an object of biological diversity of the environment, an invaluable recreational resource, and a multifaceted object of economic activity.
Fraxinus excelsior is a tree species whose natural range is spread across most European countries (Figure 1). Its geographical range extends from the Atlantic coast almost to the Volga River in the Russian Federation; its northern boundary is located in Norway at approximately 64 degrees north by latitude and extends southward to the Mediterranean through Italy and Greece. The southern limit of ash distribution in Iran is approximately at the 37-degree parallel of north latitude (Liepins et al., 2016). The natural range of $F$. excelsior is almost the same as that of the pedunculate oak (Quercus robur L.) (Dobrowolska et al., 2011).

Fraxinus excelsior is not found in the forests of central and southern Iberian Peninsula, southeastern Turkey, northern Scandinavian Peninsula and Iceland, as well as in the northern British Isles. This is primarily due to changes in environmental conditions. The northern limit of the ash range is considered to be determined by the minimum amount of solar energy, the duration

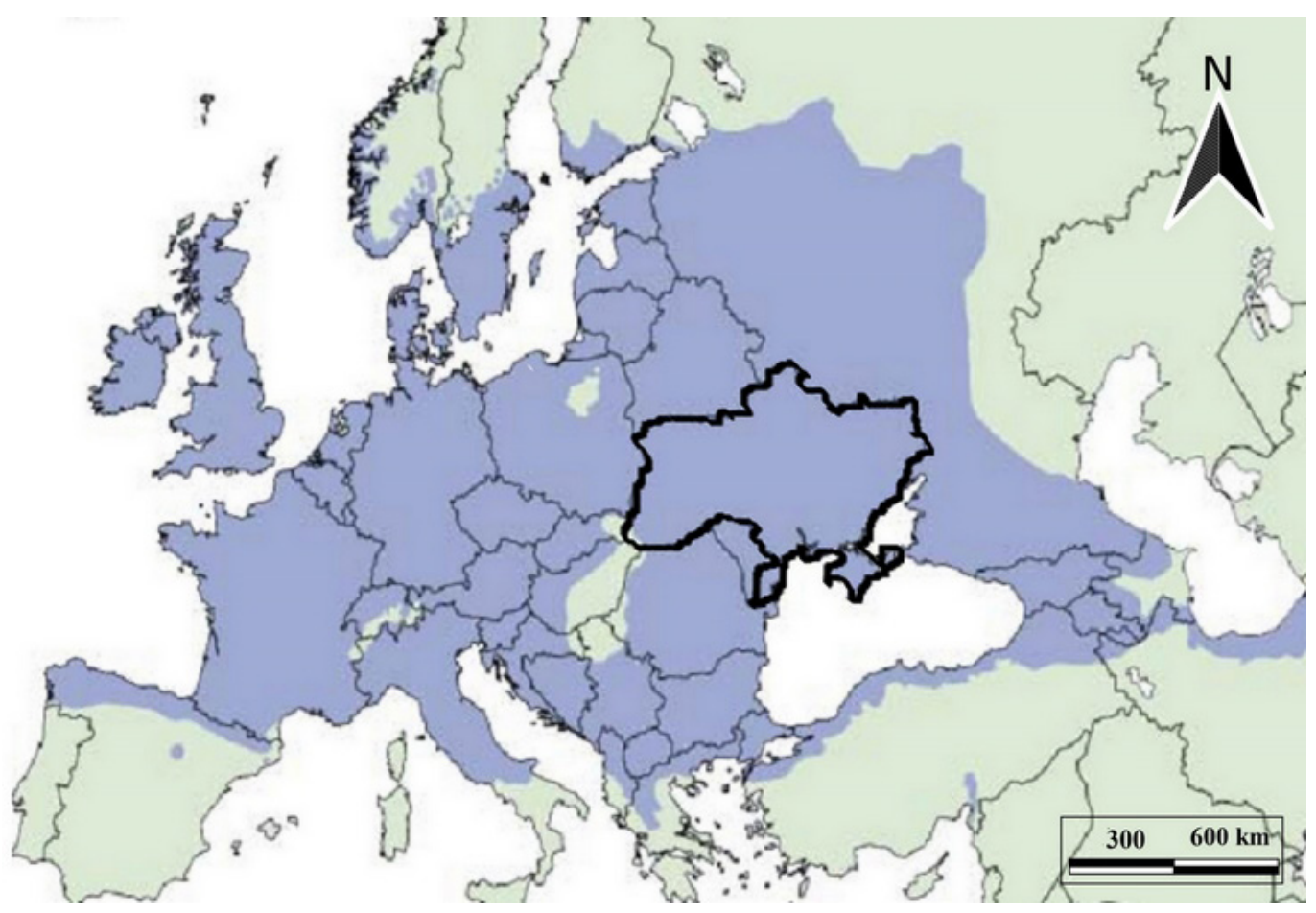

Figure 1. Natural range of common ash (Fraxinus excelsior) in Europe (Pliûra \& Heuertz, 2003) (the territory of Ukraine). 
of the growing season and the amount of heat required to complete the annual life cycle; the eastern limit is determined by the minimum temperature (up to $-15^{\circ} \mathrm{C}$ ); the southern limit depends on the deficiency in moisture. In European mountain forests, the range can reach altitudes of 1600-1800 $m$ and in northern Iran up to $2200 \mathrm{~m}$ (FRAXIGEN, 2005; Dobrowolska et al., 2011).

Common ash is an essential tree species in European deciduous forests due to its ecological characteristics, exceptional wood properties, and high economic value. It is the most valuable species of the Fraxinus genus in forestry, protective afforestation, ornamental gardening, and landscaping (Hordiyenko et al., 1996; Dobrowolska et al., 2011; Borysova, 2016; Semizer-Cuming et al., 2017). In urban landscapes, the ash value arises from its aesthetic properties such as its weeping crown shape, autumn leaf colour, attractive bark, and flowers. These trees are recommended for creating alleys, groups, single plantations in parks and urban forests, and for landscaping roads (Shvidenko \& Danilova, 2001; Percival et al., 2006).

Ash stands can regenerate in three ways: natural (seeds and stool shoots), man-made (by planting seedlings), and combined (by planting seedlings between naturally regenerated tree groups). Natural seed-regenerated ash stands contribute to the conservation of biological diversity in forests. Also, they are more resistant to adverse environmental factors, harmful insects, and diseases. Therefore, management activities in forests with common ash in the composition should ensure their natural seed regeneration.

In view of the above, we note that ash stands are of great importance for the biological diversity conservation in European, particularly in Ukranian forests. It is a valuable tree species that has long been widely used by humans, and its importance to forestry may increase in the near future (Matsiakh \& Kramarets, 2014). Promoting

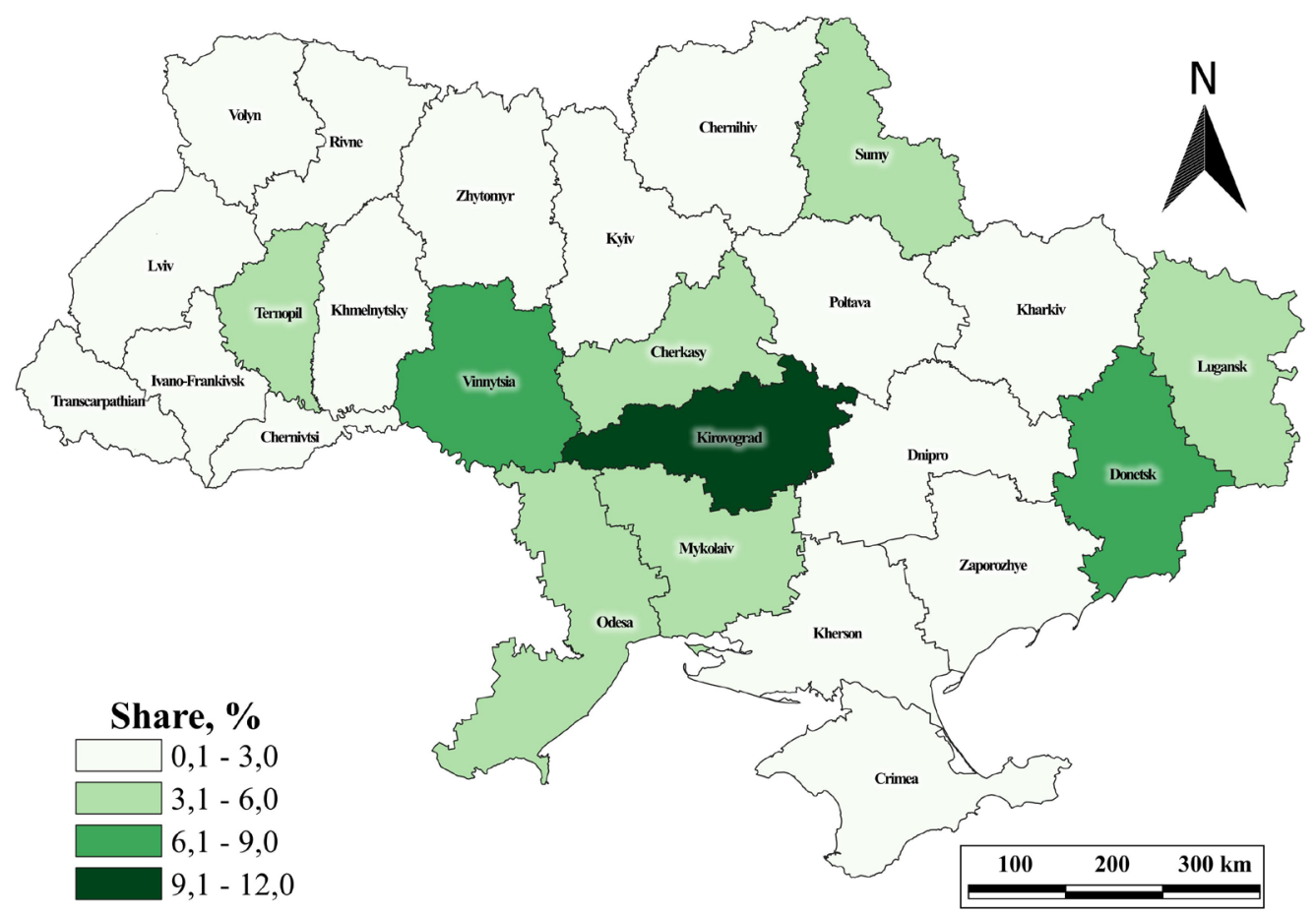

Figure 2. Common ash stands locations in Ukraine (percent of the total area). 
the natural seed regeneration of ash trees is not only an effective way of supplementing the forestation in general but also a means of increasing the sustainability of ash stands as a tree species in European forests (Enderle et al., 2017). Therefore, the main tasks for forest management in Ukraine are the conservation, sustainable use and reproduction of common ash stands.

The aim of the study was to examine the current state of ash stands in Ukraine and to identify the specificities in appearance, viability and development of common ash advanced regeneration under the canopy of oak and ash stands having different composition, age and relative density of stocking. Also, the study aimed at assessing the success of the ash natural regeneration.

\section{Materials and Methods}

\section{The study object}

The objects of the study were ash stands of different ages and relative density of stocking within 25 Ukrainian administrative regions on an area of almost 130,000 hectares (Figure 2). We studied the specificities of natural ash regeneration under the canopy of oak stands in which the ash proportion in the composition varied from 10 to $50 \%$ by stock. We also investigated pure ash stands of different ages and relative density of stocking within the specified regions.

\section{Research methodology}

The range of common ash in European forests has been specified based on the analysis of scientific studies and reference materials on the current state of Ukrainian forests. Stands with common ash trees in the composition were studied in the forests of 25 administrative regions of Ukraine. There, a total of 48 sample plots, from 0.25 to 0.8 ha each, were established. The sample plots were laid out in oak stands containing 10 to $50 \%$ of Fraxinus excelsior in the stock and in pure Fraxinus excelsior stands in the most characteristic areas.

Within each sample plot, a stand estimation was made by the total inventory method: the diameters at breast height (1.3 m) were measured using a Codimex S-1 caliper, and tree heights for 20-30 trees of the main species were determined using a Haglöf altimeter. For other tree species in the stand, heights of 10-15 trees were measured. The species composition of mixed stands was determined by the stock ratio of each of the tree species (Hrom, 2007).

Cores were selected by using a Pressler increment borer in 15 trees of the main species at breast height to determine the age of mature trees. The cores were air-dried, then the earlywood and latewood layers were measured to an accuracy of $0.01 \mathrm{~mm}$ using digital Henson equipment.

Natural regeneration was counted within each sample plot on 30 circular registration plots of $10 \mathrm{~m}^{2}(R=178 \mathrm{~cm})$ according to the method developed in the Ukrainian Research Institute of Forestry and Forest Melioration. The accounted natural regeneration was divided by species, age, and viability (Pasternak, 1990). The number of advance regeneration in stems per 1 ha $(N)$ was calculated by the following formula:

$$
N=\frac{n}{S} \times 10,000
$$

where $n$ is the number of advance regeneration stems on the registration plots; $S$ is the area of the registration plots, $\mathrm{m}^{2}$.

A total of 1,440 accounting sites within 48 sample plots (30 sites per a sample plot) were established in 15 administrative regions of Ukraine. In Vinnytsya Region, Volyn Region, Zaporizhzhya Region and Zhytomyr Region, one sample plot was established in oak stands and one sample plot in ash stands in each administrative region. In Cherkasy Region, Dnipropetrovsk Region, Kyiv Region, Kirovohrad Region and Luhansk Region two sample plots were laid out in oak stands and one sample plot in ash stands. In Chernihiv Region, 
Donetsk Region and Sumy Region three sample plots and one sample plot were established, respectively. In Poltava Region the numbers of sample plots were four and one, respectively, and in Kharkiv Region five and one sample plot, respectively.

Depending on the age of natural regeneration, we distinguished 1-, 2-, ..., and 8-year-old plants. The older advance regeneration was not observed under the stand canopy. The age was determined by cutting the trunk and counting the number of annual layers near the root collar. A minimum of 10 trees of each species was measured in the sample plot.

According to the viability status, the advance regeneration was divided into vigorous (healthy, with no visible signs of damage) and weak (affected by the pathogenic fungus Hymenoscyphus pseudoalbidus (anamorph Chalara fraxinea)).

To assess an even distribution of advance regeneration over the area, we used an occurrence index as a ratio of the number of registration plots with advance regeneration to the total number of registration plots under the canopy, expressed as a percentage. There are three categories of occurrence: natural advance regeneration, evenly distributed over the area (frequency of occurrence is above $65 \%$ ); natural advance regeneration unevenly distributed over the area (frequency of occurrence is $40-65 \%)$; grouped natural advance regeneration, with groups of at least 10 small or 5 medium and large vigorous regenerating plants.

The research data were processed using mathematical statistics (Atramentova \& Utevskaya, 2008) and MS Excel and Statistica 10 software applications. Descriptive statistics and simple pair regression analysis were used.

\section{Results}

Within Ukraine, the largest areas of forests with common ash predominating in the com- position are in Vinnytsya (14,200 ha), Sumy (12,800 ha), Luhansk (12,300 ha), Kirovohrad (11,900 ha), and Cherkasy (11,500 ha) administrative regions, and the smallest ones are in Kherson (100 ha), Zaporizhzhya (700 ha), Ivano-Frankivsk (1,300 ha), Chernivtsi (1,400 ha), and Dnipropetrovsk (1,500 ha) regions. Therefore, the largest areas of ash stands are found in the eastern and central regions of Ukraine and the smallest ones in the western, northern and southern regions. In general, in Ukraine, Fraxinus excelsior stands cover 130,000 ha; their average age is 59 years (Table 1 ).

In Ukrainian forests within the studied regions, ash mainly grows in mixed stands with other deciduous tree species. It sometimes prevails in the species composition, forming pure ash stands, but most commonly occurs as an additional species in oak stands (Spiecker et al., 2009; Vacek et al., 2015; Bachynska, 2017) (Table 2).

Only $6.4 \%$ (8,300 ha) of the total area of common ash forests in the country $(130,000$ ha) is occupied by pure ash stands. The rest $(93.6 \%$ or $121,700 \mathrm{ha})$ are mixed stands, with $40 \%$ (26,900 ha), $50 \%$ (24,600 ha), $60 \%$ (20,000 ha), and 70\% (16,300 ha) of ash. The smallest area - only 1,200 ha - is occupied by stands having $20 \%$ and less ash in the composition.

Specificities of common ash natural regeneration in the studied region were investigated under the canopy of mixed oak and ash stands, as well as in pure ash stands of different age and relative density of stocking. When assessing the condition of stands including ash, it is necessary to consider the abundance of advance regeneration and its condition. Ash has a high regenerative capacity, producing both many seeds and coppice shoots (Table 3 ). In the surveyed plots there was also natural advance regeneration of other tree species, such as Tília cordata Mill., Acer platanoides L., Acer campestre L., Quercus robur L., Ulmus glabra Huds., Populus tremula L., the number of which in some areas was $80-90 \%$ of the total regeneration number. 
Table 1. Current state of common ash (Fraxinus excelsior) stands in Ukraine (as of 2016).

\begin{tabular}{|c|c|c|c|c|}
\hline \multirow[b]{2}{*}{ Administative Regions } & \multirow{2}{*}{$\begin{array}{l}\text { Total forest area } \\
\text { (thousand ha) }\end{array}$} & \multicolumn{3}{|c|}{ Including common ash stands } \\
\hline & & thousand ha & $\begin{array}{l}\text { percent of the } \\
\text { total area }\end{array}$ & $\begin{array}{c}\text { average age } \\
\text { (years) }\end{array}$ \\
\hline Autonomous Republic of Crimea & 227.7 & 4.8 & 2.1 & 89 \\
\hline Cherkasy Region & 255.4 & 11.5 & 4.5 & 55 \\
\hline Chernihiv Region & 355.5 & 3.0 & 0.8 & 44 \\
\hline Chernivtsi Region & 157.3 & 1.4 & 0.9 & 55 \\
\hline Dnipropetrovsk Region & 65.7 & 1.5 & 2.3 & 64 \\
\hline Donetsk Region & 92.5 & 7.2 & 7.7 & 69 \\
\hline Ivano-Frankivsk Region & 425.8 & 1.3 & 0.3 & 46 \\
\hline Kharkiv Region & 282.3 & 7.1 & 2.5 & 57 \\
\hline Kherson Region & 77.3 & 0.1 & 0.1 & 63 \\
\hline Khmelnytsky Region & 167.4 & 5.1 & 3.0 & 63 \\
\hline Kyiv Region & 355.1 & 6.1 & 1.7 & 54 \\
\hline Kirovohrad Region & 103.5 & 11.9 & 11.5 & 56 \\
\hline Luhansk Region & 228.2 & 12.3 & 5.4 & 60 \\
\hline Lviv Region & 428.2 & 3.9 & 0.9 & 55 \\
\hline Mykolayiv Region & 37.3 & 1.5 & 4.0 & 59 \\
\hline Odessa Region & 90.2 & 4.5 & 5.0 & 65 \\
\hline Poltava Region & 157.3 & 3.1 & 2.0 & 53 \\
\hline Rivne Region & 584.2 & 2.4 & 0.4 & 53 \\
\hline Sumy Region & 255.6 & 12.8 & 5.0 & 57 \\
\hline Ternopil Region & 143.6 & 6.4 & 4.5 & 58 \\
\hline Transcarpathian Region & 461.2 & 2.6 & 0.6 & 54 \\
\hline Vinnytsya Region & 199.7 & 14.2 & 7.1 & 67 \\
\hline Volyn Region & 436.8 & 1.7 & 0.4 & 46 \\
\hline Zaporizhzhya Region & 34.0 & 0.7 & 2.1 & 46 \\
\hline Zhytomyr Region & 660.3 & 2.9 & 0.4 & 50 \\
\hline Total Ukraine & $6,282.2$ & 130.0 & 2.1 & 59 \\
\hline
\end{tabular}


Table 2. Distribution of the ash area in Ukraine by administrative regions in terms of ash proportion (percent of the stock) in mixed stands.

\begin{tabular}{|c|c|c|c|c|c|c|c|c|c|c|}
\hline \multirow{3}{*}{$\begin{array}{l}\text { Administative } \\
\text { Regions }\end{array}$} & \multicolumn{10}{|c|}{ Area of ash stands (numerator - thousand ha, denominator - percent of the area) } \\
\hline & \multirow{2}{*}{ Total } & \multicolumn{9}{|c|}{ Including by ash proportion (percent of the stock) in mixed stands } \\
\hline & & $\leq 20$ & 30 & 40 & 50 & 60 & 70 & 80 & 90 & 100 \\
\hline $\begin{array}{l}\text { Autonomous } \\
\text { Republic of Crimea }\end{array}$ & $\frac{4.8}{100}$ & - & $\frac{0.2}{4.2}$ & $\frac{0.5}{10.4}$ & $\frac{1.0}{20.8}$ & $\frac{1.3}{27.2}$ & $\frac{0.7}{14.6}$ & $\frac{0.5}{10.4}$ & $\frac{0.3}{6.2}$ & $\frac{0.3}{6.2}$ \\
\hline Cherkasy Region & $\frac{11.5}{100}$ & $\frac{0.1}{0.9}$ & $\frac{1.6}{13.9}$ & $\frac{3.2}{27.8}$ & $\frac{2.6}{22.6}$ & $\frac{1.8}{15.6}$ & $\frac{0.9}{7.8}$ & $\frac{0.5}{4.4}$ & $\frac{0.3}{2.6}$ & $\frac{0.5}{4.4}$ \\
\hline Chernihiv Region & $\begin{array}{l}3.0 \\
100\end{array}$ & - & $\frac{0.8}{26.7}$ & $\frac{0.8}{26.7}$ & $\frac{0.6}{20.0}$ & $\frac{0.4}{13.4}$ & $\frac{0.2}{6.6}$ & $\frac{0.1}{3.3}$ & $\frac{0.1}{3.3}$ & - \\
\hline Chernivtsi Region & $\frac{1.4}{100}$ & - & $\frac{0.1}{7.1}$ & $\frac{0.3}{21.5}$ & $\frac{0.3}{21.5}$ & $\frac{0.2}{14.3}$ & $\frac{0.2}{14.3}$ & $\frac{0.1}{7.1}$ & $\frac{0.1}{7.1}$ & $\frac{0.1}{7.1}$ \\
\hline $\begin{array}{l}\text { Dnipropetrovsk } \\
\text { Region }\end{array}$ & $\frac{1.5}{100}$ & - & $\frac{0.1}{6.7}$ & $\frac{0.3}{20.0}$ & $\frac{0.3}{20.0}$ & $\frac{0.1}{6.7}$ & $\frac{0.2}{13.3}$ & $\frac{0.3}{20.0}$ & $\frac{0.1}{6.7}$ & $\frac{0.1}{6.6}$ \\
\hline Donetsk Region & $\frac{7.1}{100}$ & - & $\frac{0.1}{1.4}$ & $\frac{0.3}{4.2}$ & $\frac{0.4}{5.6}$ & $\frac{1.3}{18.3}$ & $\frac{1.6}{22.5}$ & $\frac{1.9}{26.8}$ & $\frac{0.8}{11.3}$ & $\frac{0.7}{9.9}$ \\
\hline $\begin{array}{l}\text { Ivano-Frankivsk } \\
\text { Region }\end{array}$ & $\frac{1.3}{100}$ & - & $\frac{0.1}{7.7}$ & $\frac{0.2}{15.4}$ & $\frac{0.2}{15.4}$ & $\frac{0.3}{23.0}$ & $\frac{0.2}{15.4}$ & $\frac{0.1}{7.7}$ & $\frac{0.1}{7.7}$ & $\frac{0.1}{7.7}$ \\
\hline Kharkiv Region & $\frac{7.1}{100}$ & - & $\frac{0.3}{4.2}$ & $\frac{1.1}{15.5}$ & $\frac{1.5}{21.2}$ & $\frac{1.4}{19.8}$ & $\frac{1.2}{16.9}$ & $\frac{0.8}{11.2}$ & $\frac{0.4}{5.6}$ & $\frac{0.4}{5.6}$ \\
\hline Kherson Region & $\frac{0.1}{100}$ & - & $\frac{0.01}{10.0}$ & $\frac{0.01}{10.0}$ & $\frac{0.01}{10.0}$ & $\frac{0.01}{10.0}$ & $\frac{0.01}{10.0}$ & $\frac{0.02}{20.0}$ & $\frac{0.01}{10.0}$ & $\frac{0.01}{10.0}$ \\
\hline Khmelnytsky Region & $\frac{5.1}{100}$ & $\frac{0.2}{3.9}$ & $\frac{0.5}{9.8}$ & $\frac{1.5}{29.4}$ & $\frac{1.2}{23.6}$ & $\frac{0.8}{15.7}$ & $\frac{0.4}{7.8}$ & $\frac{0.2}{3.9}$ & $\frac{0.1}{2.0}$ & $\frac{0.2}{3.9}$ \\
\hline Kyiv Region & $\begin{array}{l}6.1 \\
100\end{array}$ & $\frac{0.3}{4.9}$ & $\frac{1.5}{24.6}$ & $\frac{1.7}{27.9}$ & $\frac{1.1}{18.0}$ & $\frac{0.6}{9.8}$ & $\frac{0.3}{4.9}$ & $\frac{0.3}{4.9}$ & $\frac{0.1}{1.7}$ & $\frac{0.2}{3.3}$ \\
\hline Kirovohrad Region & $\frac{11.9}{100}$ & - & $\frac{0.3}{2.5}$ & $\frac{1.1}{9.2}$ & $\frac{1.5}{12.6}$ & $\frac{1.4}{11.8}$ & $\frac{2.0}{16.8}$ & $\frac{2.2}{18.5}$ & $\frac{1.4}{11.8}$ & $\frac{2.0}{16.8}$ \\
\hline Luhansk Region & $\frac{12.3}{100}$ & - & $\frac{0.2}{1.6}$ & $\frac{1.4}{11.4}$ & $\frac{2.1}{17.1}$ & $\frac{2.3}{18.7}$ & $\frac{2.5}{20.3}$ & $\frac{1.8}{14.6}$ & $\frac{0.8}{6.5}$ & $\frac{1.2}{9.8}$ \\
\hline Lviv Region & $\frac{3.9}{100}$ & $\frac{0.1}{2.5}$ & $\frac{0.6}{15.6}$ & $\frac{1.0}{25.6}$ & $\frac{0.6}{15.6}$ & $\frac{0.5}{12.7}$ & $\frac{0.4}{10.2}$ & $\frac{0.3}{7.6}$ & $\frac{0.2}{5.1}$ & $\frac{0.2}{5.1}$ \\
\hline Mykolayiv Region & $\frac{1.5}{100}$ & - & - & $\frac{0.1}{6.7}$ & $\frac{0.1}{6.7}$ & $\frac{0.2}{13.3}$ & $\frac{0.3}{20.0}$ & $\frac{0.3}{20.0}$ & $\frac{0.2}{13.3}$ & $\frac{0.3}{20.0}$ \\
\hline Odessa Region & $\frac{4.5}{100}$ & - & $\frac{0.1}{2.2}$ & $\frac{0.2}{4.4}$ & $\frac{0.3}{6.7}$ & $\frac{0.5}{11.1}$ & $\frac{1.0}{22.2}$ & $\frac{0.9}{20.0}$ & $\frac{0.7}{15.6}$ & $\frac{0.8}{17.8}$ \\
\hline Poltava Region & $\frac{3.1}{100}$ & - & $\frac{0.2}{6.4}$ & $\frac{0.6}{19.3}$ & $\frac{0.6}{19.3}$ & $\frac{0.7}{22.7}$ & $\frac{0.4}{13.0}$ & $\frac{0.3}{9.7}$ & $\frac{0.1}{3.2}$ & $\frac{0.2}{6.4}$ \\
\hline Rivne Region & $\frac{2.4}{100}$ & - & $\frac{0.4}{16.7}$ & $\frac{0.7}{29.1}$ & $\frac{0.5}{20.7}$ & $\frac{0.4}{16.7}$ & $\frac{0.1}{4.2}$ & $\frac{0.1}{4.2}$ & $\frac{0.1}{4.2}$ & $\frac{0.1}{4.2}$ \\
\hline Sumy Region & $\frac{12.8}{100}$ & $\frac{0.1}{0.8}$ & $\frac{1.2}{9.4}$ & $\frac{3.1}{24.2}$ & $\frac{3.6}{28.1}$ & $\frac{2.4}{18.8}$ & $\frac{1.4}{10.9}$ & $\frac{0.6}{4.6}$ & $\frac{0.2}{1.6}$ & $\frac{0.2}{1.6}$ \\
\hline Ternopil Region & $\frac{6.4}{100}$ & $\frac{0.2}{3.1}$ & $\frac{1.3}{20.3}$ & $\frac{1.8}{28.1}$ & $\frac{1.1}{17.1}$ & $\frac{0.7}{10.9}$ & $\frac{0.6}{9.4}$ & $\frac{0.3}{4.7}$ & $\frac{0.2}{3.1}$ & $\frac{0.2}{3.1}$ \\
\hline $\begin{array}{l}\text { Transcarpathian } \\
\text { Region }\end{array}$ & $\frac{2.6}{100}$ & - & $\frac{0.2}{7.7}$ & $\frac{0.5}{19.2}$ & $\frac{0.4}{15.4}$ & $\frac{0.4}{15.4}$ & $\frac{0.5}{19.2}$ & $\frac{0.2}{7.7}$ & $\frac{0.2}{7.7}$ & $\frac{0.2}{7.7}$ \\
\hline Vinnytsya Region & $\frac{14.2}{100}$ & $\frac{0.2}{1.4}$ & $\frac{2.6}{18.3}$ & $\frac{5.2}{36.6}$ & $\frac{3.4}{23.9}$ & $\frac{1.6}{11.4}$ & $\frac{0.6}{4.2}$ & $\frac{0.4}{2.8}$ & $\frac{0.1}{0.7}$ & $\frac{0.1}{0.7}$ \\
\hline Volyn Region & $\frac{1.7}{100}$ & - & $\frac{0.4}{23.5}$ & $\frac{0.5}{29.4}$ & $\frac{0.4}{23.5}$ & $\frac{0.2}{11.8}$ & $\frac{0.1}{5.9}$ & $\frac{0.1}{5.9}$ & - & - \\
\hline
\end{tabular}


Table 2 continues

\begin{tabular}{|c|c|c|c|c|c|c|c|c|c|c|}
\hline \multirow{3}{*}{$\begin{array}{l}\text { Administative } \\
\text { Regions }\end{array}$} & \multicolumn{10}{|c|}{ Area of ash stands (numerator - thousand ha, denominator - percent of the area) } \\
\hline & \multirow{2}{*}{ Total } & \multicolumn{9}{|c|}{ Including by ash proportion (percent of the stock) in mixed stands } \\
\hline & & $\leq 20$ & 30 & 40 & 50 & 60 & 70 & 80 & 90 & 100 \\
\hline $\begin{array}{l}\text { Zaporizhzhya } \\
\text { Region }\end{array}$ & $\frac{0.7}{100}$ & - & - & $\frac{0.1}{14.3}$ & $\frac{0.1}{14.3}$ & $\frac{0.1}{14.3}$ & $\frac{0.1}{14.3}$ & $\frac{0.1}{14.3}$ & $\frac{0.1}{14.3}$ & $\frac{0.1}{14.2}$ \\
\hline Zhytom & $\frac{2.9}{100}$ & - & $\frac{0.6}{20.8}$ & $\frac{0.6}{20.7}$ & $\underline{0.6}$ & $\frac{0.4}{13.8}$ & $\frac{0.4}{13.8}$ & $\frac{0.1}{3.4}$ & $\frac{0.1}{3.4}$ & $\frac{0.1}{3.4}$ \\
\hline Total Ukraine & $\frac{130.0}{100}$ & $\frac{1.2}{0.9}$ & $\frac{13.4}{10.3}$ & $\frac{26.9}{20.7}$ & $\frac{24.6}{18.9}$ & $\frac{20.0}{15.4}$ & $\frac{16.3}{12.6}$ & $\frac{12.5}{9.6}$ & $\frac{6.8}{5.2}$ & $\frac{8.3}{6.4}$ \\
\hline
\end{tabular}

Table 3. Characteristics of natural advance regeneration (up to 8 years old) under the canopy of mixed oak and ash stands and pure ash stands within Ukraine (sample plots are ordered by increasing the stand age).

\begin{tabular}{|c|c|c|c|c|c|c|c|c|c|}
\hline \multirow[b]{3}{*}{$\begin{array}{l}\text { Sample } \\
\text { plot } \\
\text { number* }\end{array}$} & \multicolumn{3}{|c|}{ Characteristic of mother stands } & \multicolumn{6}{|c|}{$\begin{array}{l}\text { The number of advance reproduction } \\
\text { (thousand stems ha-1) }\end{array}$} \\
\hline & \multirow[b]{2}{*}{ Composition, $\% * *$} & \multirow[b]{2}{*}{ 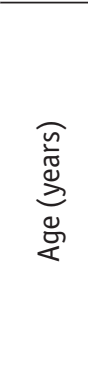 } & \multirow[b]{2}{*}{ 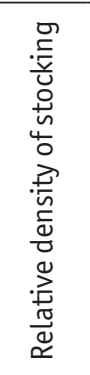 } & \multirow[b]{2}{*}{$\underset{\mathbb{0}}{\overrightarrow{0}}$} & \multicolumn{5}{|c|}{ Including common ash } \\
\hline & & & & & 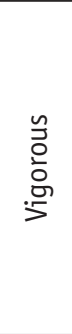 & 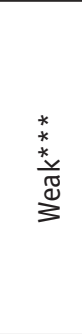 & 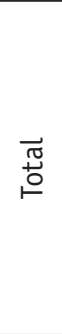 & 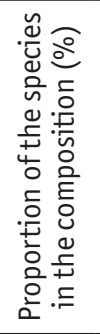 & 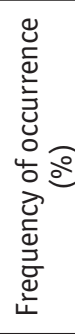 \\
\hline \multicolumn{10}{|c|}{ Cherkasy Region } \\
\hline $9-\mathrm{Ch}_{1}$ & Oak 70\%-Ash 20\%-N-Maple 10\% & 158 & 0.55 & 34.7 & 6.5 & 0.1 & 6.6 & 19 & 80 \\
\hline $3-\mathrm{Ch}_{1}$ & 0ak 50\%-Ash 30\%-Lime $20 \%$ & 188 & 0.69 & 25.0 & 7.4 & 0.5 & 7.9 & 32 & 70 \\
\hline $28-\mathrm{Ch}_{1}$ & Ash $100 \%$ & 51 & 0.83 & 4.0 & 1.0 & 0.1 & 1.1 & 27 & 40 \\
\hline \multicolumn{10}{|c|}{ Chernihiv Region } \\
\hline $7-\mathrm{Ch}_{2}$ & Oak 40\%-Ash 40\%-N-Maple $20 \%$ & 60 & 0.60 & 8.3 & 2.2 & 0.1 & 2.3 & 28 & 60 \\
\hline $11-\mathrm{Ch}_{2}$ & Oak $80 \%$-Ash $10 \%$-Lime $10 \%$ & 109 & 0.58 & 16.0 & 3.8 & 0.4 & 4.2 & 26 & 60 \\
\hline $8-\mathrm{Ch}_{2}$ & $\begin{array}{l}\text { Ash 50\%-0ak 20\%- } \\
\text { N-Maple 20\%-Lime 10\% }\end{array}$ & 119 & 0.74 & 10.2 & 1.5 & - & 1.5 & 15 & 50 \\
\hline $31-\mathrm{Ch}_{2}$ & Ash $100 \%$ & 59 & 0.78 & 4.2 & 1.3 & 0.2 & 1.5 & 36 & 50 \\
\hline \multicolumn{10}{|c|}{ Dnipropetrovsk Region } \\
\hline 22-Dn & 0ak 70\%-Ash 30\% & 91 & 0.73 & 4.4 & 0.7 & 0.7 & 1.4 & 32 & 50 \\
\hline 20-Dn & Oak $90 \%$-Ash $10 \%$ & 111 & 0.74 & 6.9 & 1.6 & 1.6 & 3.2 & 46 & 80 \\
\hline 36-Dn & Ash $100 \%$ & 52 & 0.77 & 3.6 & 1.0 & 0.2 & 1.2 & 33 & 50 \\
\hline \multicolumn{10}{|c|}{ Donetsk Region } \\
\hline 23-Do & 0ak 80\%-Ash 20\% & 70 & 0.77 & 13.7 & 5.7 & 0.3 & 6.0 & 44 & 80 \\
\hline 24-Do & 0ak $90 \%$-Ash $10 \%$ & 79 & 0.69 & 13.5 & 5.1 & 0.4 & 5.5 & 41 & 80 \\
\hline 27-Do & Oak $90 \%$-Ash $10 \%$ & 110 & 0.70 & 16.0 & 6.6 & 0.4 & 7.0 & 44 & 100 \\
\hline 33-Do & Ash $100 \%$ & 91 & 0.62 & 3.7 & 0.8 & 0.1 & 0.9 & 24 & 40 \\
\hline \multicolumn{10}{|c|}{ Kyiv Region } \\
\hline $15-\mathrm{K}$ & Oak 50\%-Ash 40\%-N-Maple 10\% & 99 & 0.93 & 11.6 & 1.9 & 0.1 & 2.0 & 17 & 50 \\
\hline $12-\mathrm{K}$ & $\begin{array}{l}\text { Oak } 60 \%-\text { Lime } 20 \%- \\
\text { Ash } 10 \%-N-\text { Maple } 10 \%\end{array}$ & 100 & 0.66 & 20.1 & 5.3 & 0.3 & 5.6 & 28 & 100 \\
\hline $37-K$ & Ash $100 \%$ & 60 & 0.67 & 3.2 & 1.0 & 0.3 & 1.3 & 41 & 60 \\
\hline
\end{tabular}


Table 3 continues

\begin{tabular}{|c|c|c|c|c|c|c|c|c|c|}
\hline \multirow[b]{3}{*}{$\begin{array}{l}\text { Sample } \\
\text { plot } \\
\text { number* }\end{array}$} & \multicolumn{3}{|c|}{ Characteristic of mother stands } & \multicolumn{6}{|c|}{$\begin{array}{l}\text { The number of advance reproduction } \\
\text { (thousand stems ha-1) }\end{array}$} \\
\hline & \multirow[b]{2}{*}{ Composition, \%** } & \multirow[b]{2}{*}{ 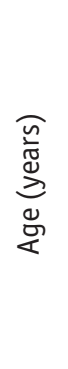 } & \multirow[b]{2}{*}{ 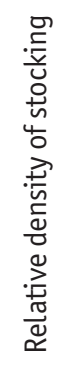 } & \multirow[b]{2}{*}{ 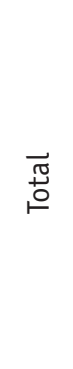 } & \multicolumn{5}{|c|}{ Including common ash } \\
\hline & & & & & $\begin{array}{l}\text { 气 } \\
\text { 옹 } \\
.00 \\
.0\end{array}$ & 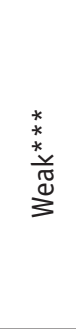 & $\underset{\stackrel{\pi}{0}}{\stackrel{0}{0}}$ & 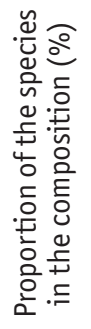 & 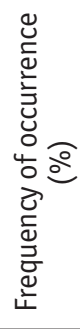 \\
\hline \multicolumn{10}{|c|}{ Kharkiv Region } \\
\hline 17-Kh & $\begin{array}{l}\text { Oak } 60 \%-\mathrm{N} \text {-Maple } 20 \%- \\
\text { Lime } 10 \% \text {-Ash } 10 \%\end{array}$ & 84 & 0.74 & 25.9 & 8.0 & 0.6 & 8.6 & 33 & 100 \\
\hline $10-\mathrm{Kh}$ & Oak $80 \%$-Ash 10\%-Lime 10\% & 97 & 0.85 & 13.7 & 1.1 & 0.4 & 1.5 & 11 & 40 \\
\hline $5-\mathrm{Kh}$ & Oak 90\%-Ash 10\% & 100 & 0.50 & 11.5 & 6.4 & 1.2 & 7.6 & 66 & 100 \\
\hline $16-\mathrm{Kh}$ & $\begin{array}{l}\text { Oak } 60 \%-\text {-Lime } 20 \%- \\
\text { Ash } 10 \%-N-\text { Maple } 10 \%\end{array}$ & 134 & 0.86 & 16.0 & 5.3 & 0.4 & 5.7 & 36 & 100 \\
\hline 4-Kh & Oak 90\%-Ash 10\% & 193 & 0.69 & 26.8 & 6.4 & 0.8 & 7.2 & 27 & 80 \\
\hline $30-\mathrm{Kh}$ & Ash $100 \%$ & 77 & 0.70 & 4.8 & 2.6 & 0.1 & 2.7 & 56 & 90 \\
\hline \multicolumn{10}{|c|}{ Kirovohrad Region } \\
\hline $40-K i$ & Oak 90\%-Ash 10\% & 79 & 0.79 & 10.5 & 3.9 & 0.2 & 4.1 & 39 & 90 \\
\hline $39-K i$ & Oak 70\%-Lime 20\%-Ash 10\% & 94 & 0.72 & 19.9 & 9.2 & 0.6 & 9.8 & 49 & 100 \\
\hline $38-\mathrm{Ki}$ & Ash $100 \%$ & 59 & 0.76 & 4.6 & 1.5 & 0.2 & 1.7 & 37 & 60 \\
\hline \multicolumn{10}{|c|}{ Luhansk Region } \\
\hline $25-\mathrm{L}$ & Oak $80 \%-$ Ash $20 \%$ & 85 & 0.76 & 11.4 & 4.9 & 0.1 & 5.0 & 44 & 90 \\
\hline $26-\mathrm{L}$ & Oak 80\%-Ash 10\%-N-Maple 10\% & 92 & 0.70 & 18.3 & 7.6 & 0.7 & 8.3 & 45 & 100 \\
\hline $34-\mathrm{L}$ & Ash $100 \%$ & 42 & 0.71 & 4.3 & 0.5 & 0.2 & 0.7 & 16 & 40 \\
\hline \multicolumn{10}{|c|}{ Odesa Region } \\
\hline $42-0$ & Oak $80 \%-$ Ash $20 \%$ & 65 & 0.80 & 5.8 & 1.6 & 0.1 & 1.7 & 29 & 50 \\
\hline $41-0$ & Ash $100 \%$ & 65 & 0.74 & 4.0 & 1.1 & 0.1 & 1.2 & 30 & 50 \\
\hline \multicolumn{10}{|c|}{ Poltava Region } \\
\hline $1-P$ & Oak 70\%-Ash 20\%-Lime 10\% & 86 & 0.67 & 16.5 & 3.9 & 0.2 & 4.1 & 25 & 50 \\
\hline $19-P$ & Oak $80 \%-$ Ash $20 \%$ & 90 & 0.80 & 12.5 & 8.5 & 0.7 & 9.2 & 74 & 80 \\
\hline $21-P$ & Oak 70\%-Ash 20\%-N-Maple 10\% & 90 & 0.71 & 10.9 & 3.7 & 0.6 & 4.3 & 39 & 20 \\
\hline $2-P$ & Oak 70\%-Ash 20\%-N-Maple 10\% & 108 & 0.70 & 16.7 & 6.7 & 0.3 & 7.0 & 42 & 100 \\
\hline $32-P$ & Ash $100 \%$ & 75 & 0.72 & 5.3 & 2.2 & 0.2 & 2.4 & 45 & 80 \\
\hline \multicolumn{10}{|c|}{ Sumy Region } \\
\hline $13-S$ & $\begin{array}{l}\text { Oak } 50 \% \text {-Ash } 20 \% \text {-Lime } 20 \%- \\
\text { N-Maple } 10 \%\end{array}$ & 78 & 0.77 & 35.7 & 5.1 & 0.1 & 5.2 & 15 & 100 \\
\hline $14-S$ & Oak 70\%-Lime 20\%-Ash 10\% & 79 & 0.80 & 6.5 & 1.6 & 0.1 & 1.7 & 26 & 70 \\
\hline $18-S$ & Oak 40\%-N-Maple 40\%-Ash 20\% & 109 & 0.78 & 46.1 & 32.6 & 1.5 & 34.1 & 74 & 100 \\
\hline $29-S$ & Ash $100 \%$ & 104 & 0.52 & 3.1 & 0.8 & 0.3 & 1.1 & 35 & 50 \\
\hline \multicolumn{10}{|c|}{ Vinnytsya Region } \\
\hline $44-V i$ & Oak 80\%-Ash 10\%-Lime 10\% & 103 & 0.65 & 14.4 & 1.3 & 0.2 & 1.5 & 10 & 50 \\
\hline $43-\mathrm{Vi}$ & Ash $100 \%$ & 76 & 0.69 & 4.4 & 1.3 & 0.2 & 1.5 & 34 & 60 \\
\hline \multicolumn{10}{|c|}{ Volyn Region } \\
\hline $45-V i$ & Oak 90\%-Ash 10\% & 110 & 0.65 & 17.4 & 1.0 & - & 1.0 & 6 & 40 \\
\hline $45-V i$ & Ash $100 \%$ & 45 & 0.75 & 2.4 & 0.3 & - & 0.3 & 12 & 30 \\
\hline
\end{tabular}


Table 3 continues

Characteristic of mother stands

The number of advance reproduction (thousand stems ha-1)

\begin{tabular}{|c|c|c|c|c|c|c|c|c|c|}
\hline \multirow[b]{2}{*}{$\begin{array}{l}\text { Sample } \\
\text { plot } \\
\text { number* }\end{array}$} & \multirow[b]{2}{*}{ Composition, \%** } & \multirow[b]{2}{*}{ 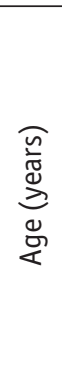 } & \multirow[b]{2}{*}{ 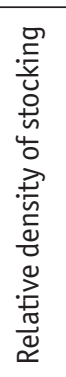 } & \multirow[b]{2}{*}{$\begin{array}{l}\overrightarrow{0} \\
\stackrel{0}{0} \\
\circ\end{array}$} & \multicolumn{5}{|c|}{ Including common ash } \\
\hline & & & & & 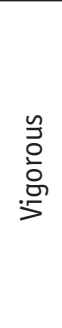 & $\begin{array}{l}* \\
* \\
* \\
\stackrel{*}{*} \\
\stackrel{\Xi}{\Xi}\end{array}$ & $\begin{array}{l}\text { त्ञ̃ } \\
\stackrel{0}{0}\end{array}$ & 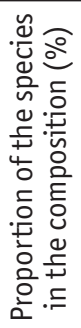 & 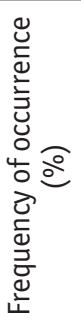 \\
\hline \multicolumn{10}{|c|}{ Zaporizhzhya Region } \\
\hline $6-Z a$ & 0ak $80 \%$-Ash $20 \%$ & 70 & 0.60 & 14.8 & 5.4 & 0.2 & 5.6 & 38 & 80 \\
\hline $35-\mathrm{Za}$ & Ash $100 \%$ & 54 & 0.50 & 2.3 & 1.1 & 0.1 & 1.2 & 52 & 50 \\
\hline \multicolumn{10}{|c|}{ Zhytomyr Region } \\
\hline 47-Zh & Oak $80 \%$-Ash $20 \%$ & 85 & 0.80 & 10.9 & 1.4 & - & 1.4 & 13 & 60 \\
\hline $46-Z h$ & Ash $100 \%$ & 50 & 0.70 & 1.9 & 0.4 & - & 0.4 & 21 & 40 \\
\hline
\end{tabular}

Notes: *Ch1 - Cherkasy Region; Ch2 - Chernihiv Region; Dn - Dnipropetrovsk Region; Do - Donetsk Region; K - Kyiv Region; Kh - Kharkiv Region; Ki - Kirovohrad Region; L - Luhansk Region; O - Odessa Region; P - Poltava Region; S - Sumy Region; Vi - Vinnytsya Region; Vo - Volyn Region; Za - Zaporizhzhya Region; Zh - Zhytomyr Region; ** Oak = pedunculate oak (Quercus robur L.), Ash = common ash (Fraxinus excelsior L.), N-Maple = Norway maple (Acer platanoides L.), Lime - small-leaved lime (Tilia cordata Mill.); ${ }^{* * *}$ Natural advance regeneration of ash infected by the pathogenic fungus Hymenoscyphus pseudoalbidus (anamorph Chalara fraxinea).

During 2000-2016, the total area of young ash stands increased by 26,000 hectares, mainly in fresh fertile sites as a result of the species change - common oak to common ash. The largest changes occurred in Lugansk (5,300 ha), Sumy (4,700 ha), Kirovohrad (3,800 ha), Cherkasy (2,800 ha), Kharkiv (2,100 ha), Poltava (1,600 ha), Donetsk (1,100 ha), Kyiv and Zhytomyr (1,000 ha each) Regions. In other administrative regions, changes in the area of ash stands amounted to less than 1,000 ha.

Mixed stands with 10 to $20 \%$ of ash in the stock allow for the best conditions for ash seed regeneration. In such stands, the total number of advance regeneration of all species is on average $18,400-18,000$ stems ha $^{-1}$, including 8,000-5,700 stems ha ${ }^{-1}$ of ash (Table 3, 4). In closed stands with the relative density of stocking of 0.70-0.80, the total number of advance regeneration of all species is on average 13,500-15,300 stems ha-1, including ash with 4,600-7,200 stems ha-1 (Table 3,5). There are many reasons for this number of ash in advance regeneration. One of the most important reason is that its young seedlings cannot compete with herbs.

Table 4. Average characteristics of natural regeneration of common ash in pure and mixed stands within Ukraine (by its proportion in the stand composition).

\begin{tabular}{lcccc}
\hline $\begin{array}{l}\text { Proportion } \\
\text { of common } \\
\text { ash in the } \\
\text { composition } \\
(\%)\end{array}$ & $\begin{array}{c}\text { Number } \\
\left(\text { stems ha }{ }^{-1}\right)\end{array}$ & $\begin{array}{c}\text { Percent of the total } \\
\text { number }\end{array}$ & $\begin{array}{c}\text { Proportion of vigorous } \\
\text { regeneration (\%) }\end{array}$ & $\begin{array}{c}\text { Frequency of } \\
\text { occurrence (\%) }\end{array}$ \\
\cline { 2 - 5 } 10 & $8,000 \pm 2,500$ & $39.7 \pm 5.0$ & $87.1 \pm 3.8$ & $87.5 \pm 5.4$ \\
20 & $5,700 \pm 500$ & $36.5 \pm 5.3$ & $94.6 \pm 1.2$ & $74.0 \pm 7.8$ \\
$30-50$ & $3,000 \pm 1,200$ & $24.6 \pm 3.6$ & $86.9 \pm 9.3$ & $56.0 \pm 4.0$ \\
100 & $1,400 \pm 200$ & $36.7 \pm 3.9$ & $85.0 \pm 2.7$ & $55.0 \pm 5.4$ \\
\hline
\end{tabular}


Table 5. Average characteristics of natural regeneration of common ash in pure and mixed stands within Ukraine, having various relative density of stocking.

\begin{tabular}{lcccc}
\hline \multirow{2}{*}{$\begin{array}{l}\text { Relative } \\
\text { density of } \\
\text { stocking }\end{array}$} & $\begin{array}{c}\text { Number } \\
\left(\text { stems ha }^{-1}\right)\end{array}$ & $\begin{array}{c}\text { Percent of the total } \\
\text { number }\end{array}$ & $\begin{array}{c}\text { Proportion of vigorous } \\
\text { regeneration (\%) }\end{array}$ & $\begin{array}{c}\text { Frequency of } \\
\text { occurrence }(\%)\end{array}$ \\
\hline $0.45-0.54$ & $3,300 \pm 2,100$ & $51.3 \pm 8.8$ & $82.8 \pm 5.5$ & $66.7 \pm 16.7$ \\
$0.55-0.64$ & $3,900 \pm 1,000$ & $27.0 \pm 3.1$ & $94.0 \pm 1.8$ & $64.0 \pm 7.5$ \\
$0.65-0.74$ & $4,600 \pm 700$ & $35.7 \pm 2.7$ & $86.0 \pm 3.7$ & $73.5 \pm 6.0$ \\
$0.75-0.84$ & $7,200 \pm 3,500$ & $41.4 \pm 6.8$ & $92.7 \pm 1.7$ & $73.3 \pm 7.4$ \\
$0.85-0.94$ & $3,100 \pm 1,300$ & $21.2 \pm 7.4$ & $87.1 \pm 6.9$ & $63.3 \pm 18.5$ \\
\hline
\end{tabular}

The relative density of stocking decreased to 0.50 in mixed stands which worsens the conditions for tree species regeneration, including ash. The number of natural regeneration in such stands is reduced to 5,600 stems ha-1, including ash to 3,300 stems $\mathrm{ha}^{-1}$. In pure ash stands with the relative density of stocking of $0.50-0.60$, conditions for the occurrence and development of natural regeneration are significantly worsened. The number of natural regeneration of all species is 2,300-3,700 stems ha-1, including ash with 800-1,100 stems ha-1 (see Table 3).

The highest number of natural ash regeneration was recorded under the canopy of old-aged stands (over 100 years old) 6,900-8,300 stems ha-1 (Table 6). Under the canopy of such stands, the optimal forest environment was formed for the successful natural regeneration of all species, including ash.

Table 6. Average characteristics of natural regeneration of common ash in pure and mixed stands within Ukraine depending on their age.

\begin{tabular}{lcccc}
\hline \multirow{2}{*}{$\begin{array}{l}\text { Age of } \\
\text { stands } \\
\text { (years) }\end{array}$} & $\begin{array}{c}\text { Number } \\
\left(\text { stems ha-1 }^{-1}\right)\end{array}$ & $\begin{array}{c}\text { Percent of the total } \\
\text { number }\end{array}$ & $\begin{array}{c}\text { Proportion of vigorous } \\
\text { regeneration }(\%)\end{array}$ & $\begin{array}{c}\text { Frequency of } \\
\text { occurrence }(\%)\end{array}$ \\
\cline { 2 - 5 } & $1,300 \pm 200$ & $33.3 \pm 4.3$ & $85.2 \pm 3.3$ & $50.0 \pm 3.1$ \\
$61-60$ & $4,200 \pm 700$ & $37.8 \pm 5.1$ & $94.9 \pm 0.8$ & $82.9 \pm 3.6$ \\
$81-100$ & $4,900 \pm 900$ & $36.5 \pm 5.4$ & $86.8 \pm 3.8$ & $68.3 \pm 8.5$ \\
$101-120$ & $8,300 \pm 4,400$ & $40.4 \pm 7.0$ & $85.5 \pm 6.8$ & $77.1 \pm 8.9$ \\
$\geq 121$ & $6,900 \pm 500$ & $28.3 \pm 3.6$ & $93.5 \pm 2.0$ & $82.5 \pm 6.3$ \\
\hline
\end{tabular}

\section{Discussion}

Special aspects of growth and development of ash stands in Ukraine, their ecological and forestry properties, methods of reforestation, improvement of health status and productivity of these stands as well as characterization of their state by regions have been addressed in many studies (Hordiyenko et al., 1996; Davydenko et al., 2013; Matsiakh \& Kramarets, 2014;
Davydenko \& Meshkova, 2017; Meshkova \& Borysova, 2017a, 2017b; Meshkova et al., 2018; Meshkova et al., 2019).

$F$. excelsior has been found to be one of the most common species in the deciduous forests within Left-Bank Ukraine (Davydenko \& Meshkova, 2014; Borysova, 2016; Davydenko \& Meshkova, 2017). In Kharkiv Region, mixed middle-aged ash stands with the relative density of stocking of 0.7-0.9 predominate; their site class 
is mainly I and II (Borysova, 2016). In the forests within Left-Bank Ukraine, the proportion of common ash trees with partial mortality caused by the invasive pathogen Hymenoscyphus pseudoalbidus is the largest in the steppe part of Kharkiv Region and the smallest in the forest-steppe part of Sumy Region (Davydenko et al., 2013).

Common ash quite often occurs as an additional species in oak stands. In wetter conditions, it is found in the composition of common alder stands (Alnus glutinosa (L.) Gaerth.). In oak forests, it can be considered the second main forest-forming species after pedunculate oak. Therefore, upon successful natural seed regeneration of ash, it is useful to cultivate sustainable ash stands of natural origin. Fedets (2006) also proposed to add significant numbers of common ash (20-40\%) and other valuable hardwood species both during initial and additional planting of forest crops to enrich the composition of the stands and their layering. This will increase the stand resistance to pests and diseases and ensure biological diversity of forests.

The study of climate change impacts on the ash radial increment in the forest-steppe part of Left-Bank Ukraine identified the deterioration of stands during the last decades (Koval \& Borysova, 2019) due to insufficient rainfall and high temperatures during the growing season. This study confirmed the conclusions (Lockwood \& LeBlanc, 2017) regarding the dependence of the condition of ashes on the balance between temperature and rainfall changes and the intensity of droughts during the growing season.

Analysing the investigations of domestic and foreign researchers, we established that the most important factors contributing to the successful growth and distribution of stands with ash trees in the composition are soil and hydrological conditions. As a rule, ash stands grow in lower relief areas on fresh and moist mineral-rich and well-drained soils (Marigo et al., 2000; Dobrowolska et al., 2011; Sopushynskyy,
2012). River floodplains also create favorable conditions for the regeneration of common ash (Dufour \& Piegay, 2008). However, ash is intolerant of fluctuations in groundwater levels. When it occurs, the treetops die first (Thomas, 2016).

The more fertile and moistened soils under the ash stands contribute to successful natural regeneration. The study in the forests of the Czech Republic (Střeštík \& Šamonil, 2006) showed that ash regeneration reached the highest densities (up to 6,000 stems per $400 \mathrm{~m}^{2}$ ) on medium-deep, heavy-textured decarbonized soils. The lowest density (almost 500 stems per 400 $\mathrm{m}^{2}$ ) was found on slopes fully exposed to the south with water retention capacity lower than $20 \mathrm{~mm}$. Similar results were obtained by Tabari et al. (1999) in the deciduous forests of Belgium. The highest number of ash regeneration (up to 150,000 stems ha-1) was also recorded on mull moder with a water content of $>20 \mathrm{~mm}$. The lowest number $\left(12,700\right.$ stems ha $\left.^{-1}\right)$ was found on southern slopes with a water content of $<20 \mathrm{~mm}$.

Successful regeneration of ash occurs mainly in the natural way from fallen seeds (ash-keys). The ash-keys have a flattened linear shape; their length is $3.4-4.6 \mathrm{~cm}$ and width in the wing is $0.8-1.1 \mathrm{~cm}$. The seeds are flat. The ash-keys from different trees have different sizes and weights (Bayura, 2017).

Ash trees begin to bear fruit annually at 20 to 30 years of age, however, intervals of 2-5 years are possible. The seeds, depending on the conditions and weather (wind), can spread over a distance of $100 \mathrm{~m}$. When there is no wind, the seeds are concentrated under the crowns of mother trees or at a short distance from them. The study (Semizer-Cuming et al., 2017) revealed a significant correlation $(0.71, \mathrm{p}<0.001)$ between the ash seed dispersal distance and wind speed, indicating that strong wind favours the dispersal of ash seeds over long distances.

Most of the ash-keys remain on the mother tree during the winter. Seed ger- 
mination may not occur in the year of its fall but may be delayed for 1-2 years (Kerr, 1995). The highest density of emerging ash seedlings was recorded after the harvest year under the tree canopy in northern Belgium - 220 stems per $1 \mathrm{~m}^{2}$ on average (Tabari et al., 1999).

Edaphic conditions (mechanical composition and soil depth), along with the progressive deterioration of the climate index, are factors that limit the occurrence and distribution of ash in mountainous conditions, in particular in the forests of the Ukrainian Carpathians. In these forests, under the canopy of 100-year-old ash stands, the number of ash regeneration varies from 7,500 to 69,800 stems $\mathrm{ha}^{-1}$, and its proportion in the regeneration composition ranges from 10 to $80 \%$ (Polataichuk, 1993).

Light is one of the most essential environmental factors affecting the natural regeneration of ash. The effect of light on the growth of ash trees is complex and depends primarily on the age of the trees. For example, the seedlings and juveniles are shade-tolerant, but as they develop they rapidly lose their tolerance of shade, gradually becoming more demanding. According to the study (Marigo et al., 2000), ash trees are able to regenerate successfully down to $2 \%$ of full sunlight. The researchers also found that ash can completely stop growing in height in low light conditions $(<2 \%)$. Similar results were obtained by Jaworski (1995). The researcher concluded that light conditions above $3.0 \%$ of full sunlight are favorable for the emergence and successful growth of ash seedlings. At such light level, advance regeneration of ash was recorded in all investigated plots. The study on the light effect on the growth of ash regeneration in mixed forests in northern Belgium (Tabari et al., 1999) indicated that successful height growth was observed at the average light intensity of $28 \%$ of full sunlight and almost completely stopped at less than $2 \%$.

Ash seedlings rarely appear in mixed stands (Cherkasy and Vinnytsia Regions,
Ukraine) with the relative density of stocking of 0.9-1.0 and a dense undergrowth at the light level of $2.5 \%$ of full sunlight. The numbers of seedlings of Carpinus betulus L., Tília cordata Mill., Acer platanoides L., and other shade-tolerant species are sufficient (even up to 100,000 stems ha $^{-1}$ ) under these light conditions and they develop normally (Hordiyenko et al., 1996). In mixed stands with the relative density of stocking of $0.9-1.0$ at $2.5-5.1 \%$ of full sunlight, a significant part of young ash seedlings die by the end of the growing season. Older ash regeneration (2-4 years old) survives at light levels above $5 \%$. In mountain conditions, the minimum light level at which the ash seedlings appear under the stand canopy should be $4 \%$ of full sunlight (Hordiyenko et al., 1996).

Based on the analysis of investigations (Emborg, 1998; Vacek et al., 2015), it has been found that ash trees can successfully regenerate in gaps in the maternal stand canopy. It has also been observed (Dobrowolska et al., 2011) that moderate shading has contributed to increased height growth of the seedlings due to reduced competition with undesirable herbaceous vegetation. Studies by Gardner (1975) show that ash trees in natural regeneration, when they reach the age of 3-4 years, can compete with abundant ground vegetation. The successful growth of ash regeneration may be limited by herbivores. This specificity should be taken into account when planning natural reforestation (Čermak \& Mrkva, 2006). The growth of ash regeneration is adversely affected by frosts, especially in late spring. Furthermore, repeated frosting leads to badly forked trees as a result of splitting of non-lignified trunks, which is one of the major potentially dangerous defects in the ash trunk (Savill, 1991).

The species composition of maternal stands significantly affects the establishment and further liveability of small ash regeneration. Thus, studies by Götmark $e t$ al. (2005) in southern Sweden indicate that the highest number of natural ash regener- 
ation (nearly 6,000 stems ha-1) was recorded under the canopy of mixed deciduous forests.

The identified specificities of emergence, liveability, and growth of natural advance regeneration of ash must be used for forest management in mixed old ash stands, particularly in the context of targeting their natural seed reproduction. Promoting the natural seed regeneration of ash trees can be an effective way to conserve ash trees and increase their sustainability not only in Ukraine but also in broadleaved forests in Europe.

The significant reduction in the area of ash forests over the last 20-30 years has become a matter of great concern for the European forestry sector as a whole. Many researchers (Kowalski \& Holdenrieder, 2009; Baral et al., 2014; Skovsgaard et al., 2017; Papić et al., 2018) consider the presence of the pathogenic fungus Hymenoscyphus pseudoalbidus (anamorph Chalara fraxinea) in European forests as the main cause of this phenomenon and its corresponding consequences. The fungus was first recorded in Poland in the late 20th century (the 1990s) and has spread over the continent (Gross et al., 2014). Hymenoscyphus pseudoalbidus has also been found in Ukrainian forests (Davydenko et al., 2013; Matsiakh et al., 2016). The fungus causes damage to both adult ash trees and young individuals, both natural and planted.

The study of the degree of ash regeneration damage by Hymenoscyphus fraxineus in the forests of Latvia (Pušpure et al., 2015) showed that more than $50 \%$ of the total number of natural regeneration was of very bad, bad and average quality. A further study (Pušpure et al., 2017) revealed that the degree of damage by $H$. fraxineus to the natural regeneration in pure ash stands was higher (about $20 \%$ of all ash individuals) compared to mixed ones dominated by Alnus glutinosa (L.) Gaertn. and Betula pendula Roth., where the percentage of dead ash was only $5 \%$. Also, the density of ash advance regeneration in such stands was significantly higher (ca. 7,300 $\pm 2,700$ stems ha-1) than in pure ash stands $(4,300 \pm$ 600 stems ha $^{-1}$ ).

Investigating the impact of dieback caused by $H$. fraxineus in 90 sites in the Alps (Trentino, northern Italy) in stands with different ash proportion in the composition, Giongo et al. (2017) found high levels of damage to both young and adult trees. Thus, $50.4 \%$ or 2,261 of the 4,486 surveyed individuals of $F$. excelsior regeneration (the number varied from a minimum of 12 to a maximum of 202, with an average of 54 individuals per plot) were attributed to the 2nd and 3rd classes of the level of damage (more than 5 dead branches). Then, 17.6\% or 789 individuals were dead. Young seedlings still in the herbaceous phase and older trees have shown to be more resistant to the disease pathogen.

By counting the ash seedlings and evaluating their health in permanent monitoring plots in Estonian forests in 2013 (2,367 trees) and 2015 (1,591 trees), Palm (2016) found that $53 \%$ of the total number of seedlings in 2013 and 41\% in 2015 were affected by the pathogen $H$. fraxineus. Additionally, open field regeneration was found to be more vulnerable to the disease than seedlings under a forest canopy. Matisone et al. (2019) obtained similar results in the study of young stands with ash in the composition in the forests of Latvia. Besides, the biodiversity of stands (shrub presence) and tree height are the main factors for stand resistance to $H$. fraxineus.

\section{Conclusion}

Ash stands in Ukraine cover an area of about 150,000 hectares or $2.4 \%$ of the total forest area in the country. Among them, common ash stands are almost $87 \%$. The highest number of natural regeneration of ash trees, about 7,000-8,000 stems ha ${ }^{-1}$ on average) was found under the canopy of mixed stands (with 10 to $20 \%$ of ash in the stand). The stands are over 100 years old, 
with a relative density of stocking of $0.7-$ 0.8 . They grow in fresh and moist fertile sites (rich in minerals) with well-drained soils and the light of at least $5-6 \%$ of that on an open site, and without abundant live ground cover. Ukrainian ash stands, occupying a relatively small part in the country's forest area, are extremely valuable as a source of high-quality timber and by-products. Also, they are a powerful regulator of biosphere processes and an accumulator of huge carbon deposits. Other benefits include the preservation of biological diversity and recreational value. For their conservation, it is necessary to develop a felling system aimed at increasing their resilience to adverse environmental factors and determine the optimum rotation ages and methods for final felling.

\section{References}

Atramentova, L.A., Utevskaya, O.M. 2008. Statistical Methods in Biology. (Статистические методы в биологии). Gorlovka. 148 pp. (In Russian).

Bachynska, U.O. 2017. Forest pathology state of ash tree stands on the territory of Medobory Nature Reserve. - Scientific Bulletin of UNFU, 27(9), 19-23. https://doi.org/10.15421/40270903. (In Ukrainian with English summary).

Baral, H.-O., Queloz, V., Hosoya, T. 2014. Hymenoscyphus fraxineus, the correct scientific name for the fungus causing ash dieback in Europe. - IMA Fungus, 5(1), 79-80. https:// doi:10.5598/imafungus.2014.05.01.09.

Bayura, O.M. 2017. Biometric indicators of Fraxinus excelsior $\mathrm{L}$. fruits and its ornamental forms. - Scientific Bulletin of UNFU, 27(3), 22-24. https://doi.org/10.15421/40270303. (In Ukrainian with English summary).

Boiko, S.V. 2007. Forestry of Poland. (Лісове господарство Польщі). - Forestry and Forest Melioration, 111, 35-42. (In Ukrainian).

Boiko, S.V., Kuprina, N.P., Lukjanets, V.A., Tarnopilska, O.M. 2012. The current state forestry of Greece. (Сучасний стан лісового господарства Греціï). - The Scientific Herald Works of Lugansk National Agrarian University, Series „Agricultural Sciences“, 36, 17-24. (In Ukrainian).
Borysova, V.L. 2016. Spread of European ash in forest stands of the Forest-steppe part of Kharkiv region. (Поширення ясена звичайного у лісових насадженнях лісостепової частини Харківської області). Forestry and Forest Melioration, 128, 12-19. (In Ukrainian).

Čermak, P., Mrkva, R. 2006. Effects of game on the condition and development of natural regeneration in the Vrapač National Nature Reserve (Litovelské Pomoraví). - Journal of Forest Science, 52(7), 329-336. https://doi: 10.17221/4515-JFS.

Davydenko, K., Meshkova, V. 2014. European ash (Fraxinus excelsior) dieback - situation in Europe and Ukraine. - Forestry and Landscape Gardening, 5. 19 pp.

Davydenko, K., Meshkova, V. 2017. The current situation concerning severity and causes of ash dieback in Ukraine caused by Hymenoscyphus fraxineus. - Vasaitis, R., Enderle, R. (eds.). Dieback of European Ash (Fraxinus spp.): Consequences and Guidelines for Sustainable Management. Uppsala, Swedish University og Agricultural Sciences, 220-227.

Davydenko, K.V., Meshkova, V.L., Kuznetsova, T.L. 2013. Spread of Hymenoscyphus pseudoalbidus - the pathogen of ash dieback in the leftbank Ukraine. (Поширення Hymenoscyphus pseudoalbidus - збудника всихання ясена у лівобережній Україні). Forestry and Forest Melioration, 123, 140-145. (In Ukrainian).

Dobrowolska, D., Hein, S., Oosterbaan, A., Wagner, S., Clark, J., Skovsgaard, J.P. 2011. A review of European ash (Fraxinus excelsior L.): Implications for silviculture. - Forestry, 84(2), 133-148. https://doi:10.1093/forestry/cpr001.

Domnenkov, V.A., Torchik, M.V., Zur, A.S. 2014. State of ash stands in the Republic of Belarus. (Состояние ясеневих насаджений в республике Беларусь). - Forestry and Hunting Economy, 8, 20-22. (In Russian).

Dufour, S., Piégay, H. 2008. Geomorphological controls of Fraxinus excelsior growth and regeneration in floodplain forests. - Ecology, 89(1), 205-215. https://doi:10.1890/06-1768.1.

Emborg, J. 1998. Understorey light conditions and regeneration with respect to the structural dynamics of a near-natural temperate deciduous forest in Denmark. - Forest Ecology and Management, 106, 83-95. https://doi. org/10.1016/S0378-1127(97)00299-5.

Enderle, R., Bußkamp, J., Metzler, B. 2017. Growth performance of dense natural regeneration of Fraxinus excelsior under attack of the ash dieback agent Hymenoscyphus fraxineus. Baltic Forestry, 23(1), 218-228. 
Enderle, R., Fussi, B., Lenz, H.D., Langer, G., Nagel, R., Metzler, B. 2017. Ash dieback in Germany: research on disease development, resistance and management options. - Vasaitis R., Enderle R. (eds.). Dieback of European Ash (Fraxinus spp.): Consequences and Guidelines for Sustainable Management. Uppsala, Swedish University of Agricultural Sciences, 89-105.

Fedets, I.F. 2006. Creation of valuable hardwood stands. (Створення цінних твердолистяних насаджень). - Forestry and Forest Melioration, 110, 85-88. (In Ukrainian).

FRAXIGEN. 2005. Ash Species in Europe: Biological Characteristics and Practical Guidelines for Sustainable Use. Oxford, University of Oxford, Oxford Forestry Institute. 128 pp.

Gardner, G. 1975. Light and the growth of ash. - Light as an Ecological Factor: II. The 16th Symposium of the British Ecological Society, United Kingdom, March 1974. Blackwell, Oxford, 557-563.

Giagli, K., Baar, J., Fajstavr, M., Gryc, V., Vavrčík, H. 2018. Tree-ring width and variation of wood density in Fraxinus excelsior L. and Quercus robur L. growing in floodplain forests. - BioResources, 13(1), 804-819. https://doi: 10.15376/biores.13.1.804-819.

Giongo, S., Oliveira Longa, C.-M., Dal Maso, E., Montecchio, L., Maresi, G. 2017. Evaluating the impact of Hymenoscyphus fraxineus in Trentino (Alps, Northern Italy): first investigations. - iForest, 10, 871-878. https://doi: 10.3832/ ifor2486-010.

Götmark, F., Fridman, J., Kempe, G., Norden, B. 2005. Broadleaved tree species in conifer-dominated forestry: Regeneration and limitation of saplings in southern Sweden. - Forest Ecology and Management, 214(1), 142-157. https:/ / doi. org/10.1016/j.foreco.2005.04.001.

Gross, A., Holdenrieder, O., Pautasso, M., Queloz, V., Sieber, T.N. 2014. Hymenoscyphus pseudoalbidus, the causal agent of European ash dieback. - Molecular Plant Pathology, 15(1), 5-21. https:/ / doi: 10.1111/mpp.12073.

Gulchak, V.P. 2012. State forest register of Ukraine - Results and forecasts. (Державний облік лісів України - підсумки та прогнози). - Lisovyi i Myslyvskyi Zhurnal, 2, 6-8. (In Ukrainian).

Hordiyenko, M.I., Hoychuk, A.F., Hordiyenko, N.M., Leontyak, H.P. 1996. Ash Trees in Ukraine. (Ясени в Україні). Kyiv, Sil'hosposvita. 392 pp. (In Ukrainian).

Hrom, M.M. 2007. Forest Inventory. (Лісова таксація). Lviv, RVV NLTU. 416 pp. (In Ukrainian).

Jaworski, A. 1995. Silvicultural Characteristic of Forest Trees. Kraków, Gutenberg. 237 pp.

Kerr, G. 1995. Silviculture of ash in southern England. - Forestry, 68, 63-70. https://doi. org/10.1093/forestry/68.1.63.
Koval, I.M., Borysova, V.L. 2019. Ash radial growth response to climate change in the stands of Left Bank Forest-Steppe. - Scientific Bulletin of UNFU, 29(2), 53-57. https://doi. org/10.15421/40290210. (In Ukrainian with English summary).

Kowalski, T., Holdenrieder, O. 2009. The teleomorph of Chalara fraxinea, the causal agent of ash dieback. - Forest Pathology, 39, 304-308. https://doi.org/10.1111 /j.1439-0329.2008.00589.

Liepinš, K., Liepinš, J., Matisons, R. 2016. Growth patterns and spatial distribution of common ash (Fraxinus excelsior L.) in Latvia. - Proceedings of the Latvian academy of Sciences, Section B, 70(3), 109-115. https:// doi.org/10.1515/prolas-2016-0018.

Lockwood, B.R., LeBlanc, D.C. 2017. Radial growth-climate relationships of white ash (Fraxinus americana L. Oleaceae) in the eastern United States. - The Journal of the Torrey Botanical Society, 144(3), 267-279. https:/ / doi. org/10.3159/TORREY-D-16-00022.1.

Marigo, G., Peltier, J.-P., Girel, J., Pautou, G. 2000. Success in the demographic expansion of Fraxinus excelsior L. - Trees, 15, 1-13. https:/ / doi.org/10.1007/s004680000061.

Matisone, I., Matisons, R., Jansons, A. 2019. Health condition of European ash in young stands of diverse composition. - Baltic Forestry, 25(1), 59-62.

Matsiakh, I.P., Kramarets, V.O. 2014. Declining of common ash (Fraxinus excelsior L.) in western Ukraine. (Всихання ясена звичайного (Fraxinus excelsior L.) на заході України). Scientific Bulletin of UNFU, 24(7), 67-74. (In Ukrainian).

Matsiakh, I., Solheim, H., Nagy, N.E., Hietala, A.M., Kramarets, V. 2016. Tissue-specific DNA levels and hyphal growth patterns of Hymenoscyphus fraxineus in stems of naturally infected Fraxinus excelsior saplings. - Forest Pathology, 46(3), 206-214. https://doi. org/10.1111/efp.12245.

Meshkova, V.L., Borysova, V.L. 2017a. Damage causes of European ash in the permanent sampling plots in Kharkiv region. - Forestry and Forest Melioration, 131, 179-186.

Meshkova, V.L., Borysova, V.L. 2017b. Health condition of European ash (Fraxinus excelsior L.) in the forest stands of the Forest-steppe part of Kharkov Region of Ukraine. (Санитарное состояние ясеня обыкновенного (Fraxinus excelsior L.) в лесах лесостепной части Харьковской области Украины). - Izvestia Sankt-Peterburgskoj Lesotehniceskoj Akademii, 220, 140-154. https:// doi. org/10.21266/2079-4304.2017.220. Russian). 
Meshkova, V.L., Borysova, V.L., Skrylnik, Yu.Ye., Zinchenko, O.V. 2018. European ash health condition in the Forest-steppe part of Sumy region. - Forestry and Forest Melioration, 133, 128-135. https://doi.org/10.33220/10263365.133.2018.128.

Meshkova, V., Borysova, V., Didenko, M., Nazarenko V. 2019. Incidence and severity of symptoms assigned to Fraxinus excelsior bacterial disease in the Left-bank Forest Steppe of Ukraine. - Forestry Ideas, 25(1), 171-181.

Palm, K. 2016. The condition of European ash (Fraxinus excelsior L.) in Estonia. - Master's Thesis. Tartu, Estonian University of Life Sciences. 55 pp.

Papić, S., Buriánek, V., Longauer, R., Kudláček, T., Rozsypálek, J. 2018. Phenotypic variability of Fraxinus excelsior L. and Fraxinus angustifolia Vahl under the ash dieback disease in the Czech Republic. - Journal of Forest Science, 64, 279_ 288. https:// doi.org/10.17221/30/2018-JFS.

Pasternak, P.S. (ed.). 1990. Forestry Handbook. (Справочник лесовода). Kyiv, Urozhay. 295 pp. (In Russian).

Percival, G.C., Keary, I.P., AL-Habsi, S. 2006. An assessment of the drought tolerance of Fraxinus genotypes for urban landscape plantings. - Urban Forestry and Urban Greening, 5(1), 17-27. https://doi.org/10.1016/j. ufug.2006.03.002.

Pliûra, A., Heuertz, M. 2003. Common Ash (Fraxinus excelsior L.): Technical Guidelines for Genetic Conservation and Use. Rome, EUFORGEN. 6 pp.

Polataichuk, M.D. 1993. European ash in the Ukrainian Carpathians. (Ясень в Украинских Карпатах). - Forest Science, 1, 25-34. (In Russian).

Pušpure, I., Gerra-Inohosa, L., Arhipova, N. 2015. Quality assessment of European ash Fraxinus excelsior L. genetic resource forests in Latvia. - Proceedings of the Annual $21^{\text {st }}$ International Scientific Conference: Research for Rural Development, Latvia, 2015. Jelgava, 37-43.

Pušpure, I., Matisons, R., Laivinsš, M, Gaitnieks, T., Jansons, J. 2017. Natural regeneration of common ash in young stands in Latvia. - Baltic Forestry, 23(1), 209-217.

Savill, P.S. 1991. The Silviculture of Trees used in British Forestry. Wallingford, CABI Publishing. 160 pp.

Semizer-Cuming, D., Kjær, E.D., Finkeldey, R. 2017. Gene flow of common ash (Fraxinus excelsior L.) in a fragmented landscape. PLoS ONE, 12(10), e0186757. https://doi. org/10.1371/journal.pone.0186757.
Shvidenko, A.Y., Danilova, O.M. 2001. Forest Dendrology. (Лісова дендрологія). Chernivtsi, Zelena Bukovyna. 228 pp. (In Ukrainian).

Skovsgaard, J.P., Wilhelm, G.J, Thomsen, I.M., Metzler, B., Kirisits, T., Havrdová, L., Enderle, R., Dobrowolska, D., Cleary, M., Clark, J. 2017. Silvicultural strategies for Fraxinus excelsior in response to dieback caused by Hymenoscyphus fraxineus. - Forestry: An International Journal of Forest Research, 90(4), 455-472. https:/ / doi. org/10.1093/forestry/cpx012.

Sopushynskyy, I.M. 2012. Bio-ecological and biometric features of ash (Fraxinus excelsior L.) with wavy-grained wood. - Scientific Bulletin of UNFU, 22(8), 13-19. (In Ukrainian with English summary).

Spiecker, H., Hein, S., Makkonen-Spiecker, K., Thies, M. 2009. Valuable Broadleaved Forests in Europe. Leiden, Brill. 256 pp.

State. 2016. General characteristics of forests of Ukraine. (Загальна характеристика лісів України). State Forest Resources Agency. [WWW document]. - URL http://dklg. kmu.gov.ua/forest/control/uk/publish/ article?art_id=62921. [Accessed 24 March 2020]. (In Ukrainian).

Střeštík S., Šamonil P. 2006. Ecological valence of expanding European ash (Fraxinus excelsior L.) in the Bohemian Karst (Czech Republic). Journal of Forest Science, 52, 293-305. https:/ / doi: 10.17221/4511-JFS.

Tabari, K.M., Lust, N., Zahedi, G. 1999. Growth and development of ash (Fraxinus excelsior L.) seedlings on different humus types under a closed forest canopy. - Silva Gandavensis, 64, 44-57.

Thomas, P.A. 2016. Biological flora of the British Isles: Fraxinus excelsior. - Journal of Ecology, 104, 1158-1209. https://doi: 10.1111/13652745.12566.

Vacek, S., Vacek, Z., Bulusek, D., Putalova, T., Sarginci, M., Schwarz, O., Srutka, P., Podrazsky, V., Moser, W.K. 2015. European ash (Fraxinus excelsior L.) dieback: Disintegrating forest in the mountain protected areas, Czech Republic. - Austrian Journal of Forest Science, 4, 203-223.

Wellock, M.L., Rafique, R., LaPerle, C.M., Peichl, M., Kiely, G. 2014. Changes in ecosystem carbon stocks in a grassland ash (Fraxinus excelsior) afforestation chronosequence in Ireland. - Journal of Plant Ecology, 7(5), 429438. https://doi.org/10.1093/jpe/rtt060. 